Stomatologie 2015 · 112:125

DOI 10.1007/s00715-015-0052-7

Online publiziert: 17. Juni 2015

(c) Springer-Verlag Wien 2015

CrossMark

\section{Andreas Moritz}

Klinikvorstand der Universitätszahnklinik Wien, Medizinische Universität Wien, Wien, Österreich

\title{
Schwerpunktheft Zahnklinik Wien
}

Sehr geehrte Kollegin!

Sehr geehrter Kollege!

Mit dem diesjährig stattfindenden Jubiläum „650 Jahre Universität Wien“ möchten wir auch auf die Geschichte der Universitätszahnklinik Wien zurückblicken. Wir wollen zu diesem feierlichen Anlass die Gelegenheit nutzen, Ihnen unsere Klinik vorzustellen und Ihnen Einblicke in verschiedene Forschungsschwerpunkte aller unserer Fachbereiche zu gewähren.

Die Universitätszahnklinik Wien wurde im Jahr 2004 eine $100 \%$ ige Tochtergesellschaft der damals aus der Medizinischen Fakultät der Universität Wien hervorgegangenen Medizinischen Universität Wien. Durch diesen Schritt war es möglich, eine flexible Organisationsstruktur in einem universitären Umfeld zu schaffen und somit eine optimale $\mathrm{Pa}$ tientenbetreuung gemeinsam mit einer international anerkannten universitären Ausbildung und auch einer renommierten Forschung unter einem Dach zu vereinen.

Im Ihnen vorliegenden Schwerpunktheft „Zahnklinik Wien“ finden Sie Beiträge aus bisherigen Fachbereichen der Universitätszahnklinik (Kieferorthopädie, orale Chirurgie, Prothetik, Zahnerhaltung und Parodontologie), aber auch zweier neu berufener Fachbereiche, der Kinderzahnheilkunde und der oralen Biologie.

Dr. Ambrositsch und Prof. Bantleon (Kieferorthopädie) vergleichen in einer Studie mittels einer computergestützten Messapparatur die transversalen Kräfte und Drehmomente zweier TPA-Bogentypen bei der Molarenderotation. Dr. Heuberer (orale Chirurgie) schreibt in ihrem Artikel mit Prof. Watzak, Prof. Zechner, DDr. Zauza, Prof. Ulm und Prof. Freudenthaler über Therapieentscheidungen und unterschiedliche Therapiean- sätze bei Zahnaplasien. DDr. Bristela und DDr. Skolka von der Prothetik präsentieren einen Fallbericht und erläutern die wichtigsten diagnostischen und therapeutischen Schritte in der prothetischen Rehabilitation einer Patientin mit Funktionsstörungen im Kauorgan. Ein weiterer Fallbericht mit besonderem Augenmerk auf die prothetisch-chirurgische Zusammenarbeit wird von DDr. Kotlarenko und PD DDr. Vasak präsentiert und beschreibt eine interdisziplinäre Rehabilitation mit festsitzendem Zahnersatz. Die Zahnerhaltung ist u. a. durch einen Übersichtsartikel von Dr. Dauti über CAD/CAM-Systeme mit all ihren Vor- und Nachteilen vertreten. DDr. Cvikl (Zahnerhaltung) gibt infolge ihres Auslandsaufenthaltes an der Klinik für Zahnerhaltung, Präventiv- und Kinderzahnmedizin der Universität Bern unter der Leitung von Prof. Lussi einen Überblick über dentale Erosionen und erosive Zahnhartsubstanzverluste. Prof. Rausch-Fan vertritt in ihrem Artikel über die Rolle des okklusalen Traumas in der Pathogenese der Parodontitis den Fachbereich der Parodontologie. Die neuen Fachbereiche werden die Kinderzahnheilkunde betreffend durch einen Artikel von Prof. Bekes über mundgesundheitsbezogene Lebensqualität in der Kinderzahnheilkunde zur Beurteilung oraler Erkrankungen sowie von Therapieeffekten vorgestellt. Prof. Gruber präsentiert den ebenfalls neuen Fachbereich orale Biologie mit einem Artikel über Osteozytenschaltzentralen im Knochen und mögliche Implikationen in der Zahnmedizin und beschreibt, dass Osteozyten eine Schlüsselfunktion für die Entstehung und Funktion der knochenbildenden Osteoblasten und der knochenresorbierenden Osteoklasten haben.

Das vorliegende Schwerpunktheft „Zahnklinik Wien“ bietet Ihnen somit einen Querschnitt aus den mannigfachen Projekten der unterschiedlichen Fachbereiche der Universitätszahnklinik. Ich wünsche Ihnen viel Freude beim Lesen und würde mich freuen, wenn Sie uns besuchen kommen, um sich auch persönlich ein Bild zu machen.

Ihr

Andreas Moritz

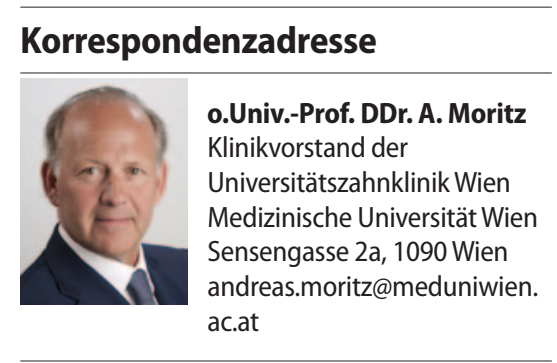

